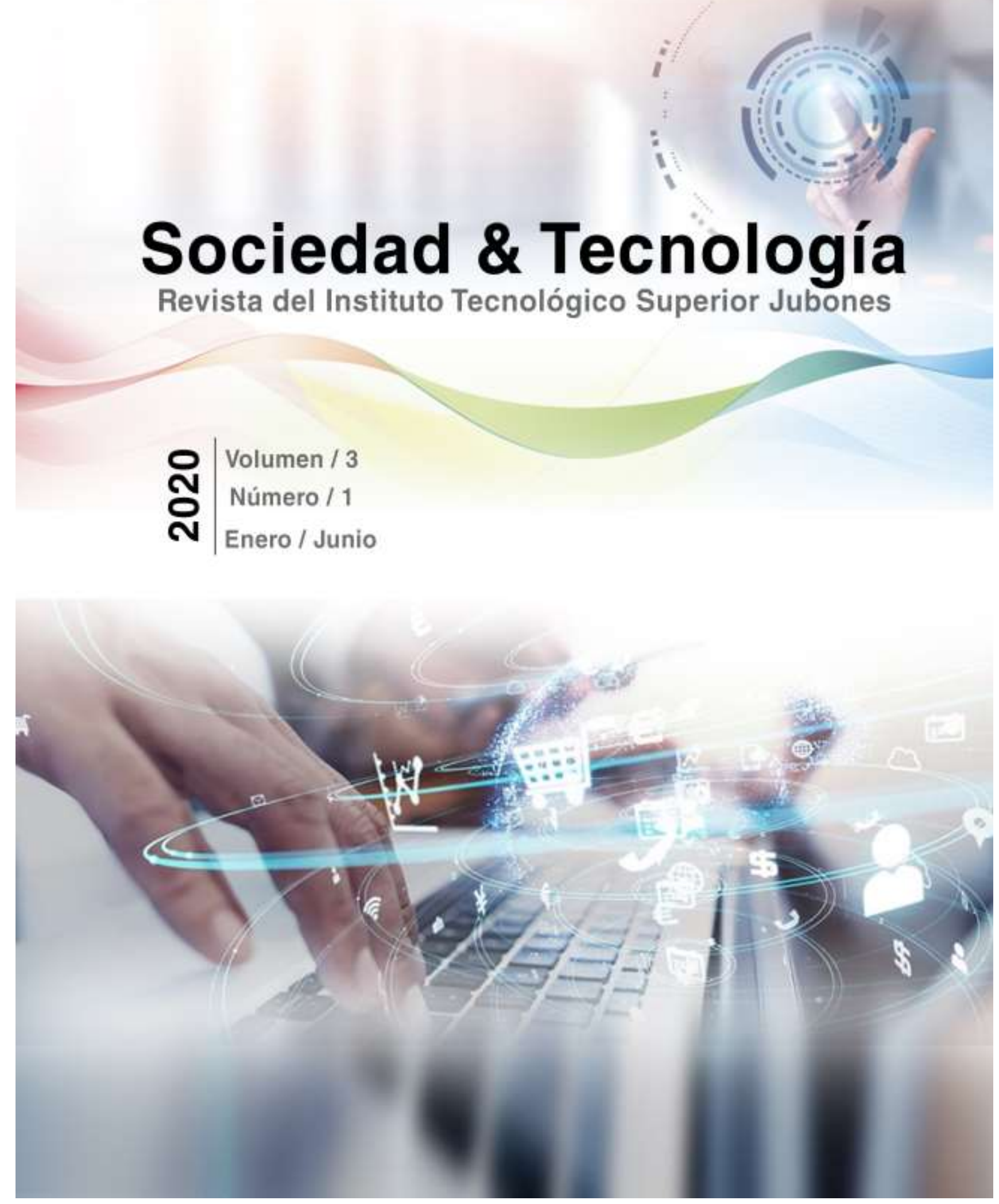




\title{
LA LABOR EDUCATIVA DE LOS DOCENTES DEL AÑO EN LA FORMACIÓN DEL PROFESIONAL DE CULTURA FÍSICA.
}

\author{
THE EDUCATIONAL WORK OF THE TEACHERS OF THE YEAR IN THE TRAINING OF THE PHYSICAL \\ CULTURE PROFESSIONAL.
}

Magda de Quesada Varona ${ }^{1}$

E-mail: mdquesadav@uo.edu.cu

ORCID: https://orcid.org/0000-0003-0587-5079

Teresa Nápoles Gómez ${ }^{1}$

E-mail: tnapolesg@uo.edu.cu

ORCID: https://orcid.org/0000-0001-5434-8641

Sulay Marrero Gorra ${ }^{1}$

E-mail: smarrerog@uo.edu.cu

ORCID: https://orcid.org/0000-0002-8847-0837

Jorge Camilo Morejón de Quesada ${ }^{1}$

E-mail: jmorejon@uo.edu.cu

ORCID: https://orcid.org/0000-0001-5500-5149

${ }^{1}$ Universidad de Oriente de Santiago de Cuba, Cuba.

Cita sugerida (APA, séptima edición)

De Quesada Varona, M., Nápoles Gómez, T., Marrero Gorra, S., \& Morejón de Quesada, J. C. (2020). La labor educativa de los docentes del año en la formación del profesional de Cultura Física. Revista Sociedad \& Tecnología, 3(1), 18-23.

\section{RESUMEN}

La presente investigación aborda una temática de importancia y actualidad en los centros de enseñanza superior referida al proceso del trabajo educativo en los estudiantes de la Licenciatura en Cultura Física. La misma revela las insuficiencias que aún se manifiestan en el proceso de formación de los estudiantes en la carrera lo cual limita su compromiso con la naturaleza pedagógica de la profesión en la práctica social. En tal sentido se aplicaron métodos de revisión y análisis documental y bibliográfico acerca del tema, los métodos empíricos para la caracterización de los grupos y los métodos de persuasión, diálogo y debate para la realización de la propuesta del trabajo educativo en la carrera Cultura Física haciendo énfasis en los elementos esenciales epistemológicos del qué, cómo y para qué de las acciones educativas en aras de incentivar en los estudiantes las obligaciones, compromiso y deber con el encargo social profesional. El aporte fundamental del trabajo se expresa en las formas, vías y métodos de organización y planificación de las acciones que persuaden, estimulan y sancionan a los estudiantes y eleva la percepción del ejemplo personal del docente.

\section{PALABRAS CLAVE:}

trabajo educativo, métodos, formación profesional

\section{ABSTRACT}


This research addresses an important and topical issue in higher education centers related to the process of educational work in students from the Sports Culture Program. It reveals the inadequacies that are still manifested in the process of training students in the career, which limits their commitment to the pedagogical nature of the profession in social practice. In this sense, methods of review and documentary and bibliographic analysis were applied on the subject, empirical methods for the characterization of the groups and the methods of persuasion, dialogue and debate for the realization of the educational work proposal in the Sports Culture career doing emphasis on the essential epistemological elements of the what, how and for what of educational actions in order to encourage in students the obligations, commitment and duty with the professional social commission. The fundamental contribution of the work is expressed in the forms, ways and methods of organization and planning of actions that persuade, stimulate and sanction students and elevate the perception of the teacher's personal example.

\section{Key words:}

educational work, methods, professional training

\section{INTRODUCCIÓN}

Un acercamiento al contexto actual de nuestro país (Cuba), nos revela una instantánea de los problemas actuales y como se están enfrentando las indisciplinas sociales, las conductas inadecuadas en todos los sentidos por lo que reclama de la labor del educador, un trabajo educativo organizado, planificado y dirigido a la transformación de la personalidad de los estudiantes universitarios, desarrollando sentimientos y convicciones; así como moldeando conductas responsables, todo lo cual se realiza con el protagonismo individual y colectivo de los estudiantes

La educación superior en Cuba asume la labor educativa de forma íntegra, con la excelencia académica, la formación de valores y el compromiso social, con un enfoque de sistema desde lo académico (curricular y extracurricular) a través de las diferentes asignaturas que lo conforman así como desde los procesos sustantivos de la extensión universitaria y la práctica laboral investigativa.
Según plantea Horruitiner (2003) en el modelo de formación las dimensiones instructiva, desarrolladora y educativa preparan con su integración al profesional para su desempeño exitoso en la sociedad, el mismo constituye un proceso complejo que se identifica como el proceso de formación de los profesionales y tiene un carácter esencialmente social que se desarrolla como un sistema en el cual están comprendidos procesos tanto de carácter curricular como extracurricular en los que se instruye, desarrolla y educa (Horruitiner, 2006)

Por lo tanto, el modelo procura dotar de los conocimientos y las habilidades esenciales de su profesión, prepararlo para emplearlas al desempeñarse en su puesto de trabajo, desarrollando en él las competencias profesionales para asegurar su desempeño laboral exitoso poniendo en práctica todo lo aprendido desde que ingresa a la Universidad hasta su graduación (Jiménez \& Peralta, 2014). Formando además en él rasgos cognitivos, afectivos y volitivos de la personalidad como profesional y como ciudadano, este proceso no puede quedar a la espontaneidad y a la inspiración de quienes intervienen en él y muchos menos de quienes lo dirigen.

Por todo lo antes planteado es necesario organizar, planificar la labor educativa de los docentes en aras de lograr que su ejecución sea exitosa y se cumplan los objetivos y metas deseadas. El trabajo educativo como concreción de la labor docente en la educación universitaria, es uno de los procesos más complejos que existen y dentro de ella misma, incluye varios factores, tales como el profesor, los alumnos, la familia y el contexto social con sus sujetos e influencias, que se interrelacionan entre sí en el proceso de formación de la personalidad como proceso social.

El trabajo educativo en la Facultad de Cultura Física de la Universidad de Oriente, se asume como el conjunto de acciones cognitivas y afectivas dirigidas a la transformación de la conciencia individual y colectiva para lograr una conducta perdurable en correspondencia con los intereses de la sociedad en una etapa histórica determinada (Báxter Pérez, 1998).

Esta conceptualización es compartida por las autoras como punto de partida, puesto que sintetiza los elementos esenciales, sin embargo dado el objeto y la problemática estudiada se defiende que la labor educativa es un proceso del hacer del docente con los estudiantes y es expresión del trabajo educativo que a su vez 
se estructura en sistema de acciones complejas, dinámicas, atemperadas al contexto histórico sociocultural existente del estudiante, que lo centra a él en el interés de las acciones educativas, sin dejar de significar el papel, la preparación del docente, su ejemplo personal, maestría pedagógica, compromisos profesionales e identidad hacia la labor $y$ responsabilidad con las tareas formativas. Luego entonces podemos afirmar que la labor educativa es una relación dialéctica entre el profesor y el alumno, donde uno y otro se complementa, y son a su vez elementos esenciales del proceso en sí mismo.

Tomando en cuenta la definición dada anteriormente y los elementos comunes a todos los autores estudiados sobre el tema del trabajo educativo, se determinan los rasgos esenciales que conforman el trabajo educativo en el año:

- Son acciones planificadas que incide en la conciencia individual $y$ colectiva.

- Se constituyen en actividades teóricas y prácticas tanto cognitivas como afectivas y volitivas.

- Implica transformación consciente del individuo $y / o$ colectivo en correspondencia con los principios e ideología del modelo social en el que se vive.

- Las propuestas de acciones y actividades parten del presupuesto de tomar en cuenta la realidad sociocultural existente, las necesidades y aspiraciones de los grupos e individuos; así como las demandas sociales técnicasprofesionales.

- Y los métodos y procedimientos elegidos para lograrla deberán estimular el libre intercambio de información brindando los suficientes argumentos que permitan juicios de valor desde el diálogo.

Por tanto, podemos afirmar que el trabajo educativo supone sembrar ideas, valores, sentimientos en los educandos, son todas aquellas acciones e influencias conscientes y dirigidas hacia un objetivo sobre el estudiante universitario, así como la organización de su actividad para enriquecer el conocimiento, en aras de formar la conciencia, desarrollar costumbres y hábitos de conducta siempre y cuando el trabajo sea sistemático y planificado en cada uno de los componentes que enriquecen a su vez la cultura universitaria de los mismos (Rojas, 2015).

Sin embargo no siempre podemos lucir resultados que muestren las mejores $y$ agradables manifestaciones de conductas, sentimientos y conocimientos de nuestros estudiantes en cuanto al cumplimiento de tareas, la solución de problemas o el desarrollo de temas e investigaciones tal como se revela en el diagnóstico de la realidad de la facultad de Cultura Física en Santiago de Cuba, en la que se muestran insuficiencias en conductas no deseadas de poca disposición y compromiso con las tareas asignadas que denotan la necesidad de continuar perfeccionando la labor educativa (Pérez, 2001).

Para dar cumplimiento a esta problemática se propone como objetivo: implementar acciones educativas en los estudiantes de la carrera Cultura Física que incentiven las obligaciones, el cumplimiento del deber y la entrega a las tareas profesionales y sociales del año desde la disciplinas.

\section{DESARROLLO}

Se analiza el Plan de estudio "E" en la carrera de Cultura Física y sus objetivos generales, de manera particular se estudian los resultados del diagnóstico de las necesidades de los estudiantes, de igual forma se consulta el proyecto educativo del año (segundo, curso diurno) y se formulan las acciones educativas que de manera integrada realizaran las asignaturas de Economía Política y Educación Física Adaptada con el propósito de formar no sólo a un estudiante que piense sino y en buena medida a un estudiante que sienta, que sea capaz de orientar su comportamiento a partir de hacer suyos, como normas y cualidades, los valores sociales más relevantes, de igual forma se vincula el contenido del aprendizaje de las asignaturas con la práctica laboral investigativa estimulando la valoración por el alumno en el plano educativo de los problemas sociales y profesionales existentes.

Las docentes realizan una dirección y un control efectivo del sistema de influencias educativas de sus alumnos mediante una planificación adecuada de las actividades y acciones educativas, ponderando la clase como la vía fundamental de lograrla y asumiendo el proyecto educativo como principal

Volumen 3 | número 1 | enero-junio | 2020 
herramienta, teniendo como elementos esenciales de su acción pedagógica el desarrollar sentimientos, formar costumbres y hábitos de conducta para lograr la formación de valores, actitudes y capacidades de autoaprendizaje en los estudiantes (Palos, 1998).

Es un proceso de mediano y largo alcance que se realiza desde el presente, tomando en cuenta el pasado y aspirando al futuro, requiere paciencia, capacidad y maestría pedagógica, parte de la motivación y el ejemplo personal de los profesores y de la correcta selección de las formas, medios y métodos para lograrlos.

Los métodos utilizados como vías para lograr el cumplimiento de los objetivos y de las acciones educativas son una compleja combinación de la actividad de los docentes y de los alumnos durante la actividad y la comunicación para garantizar la dirección de la actividad de los alumnos, estimular la asimilación teórica y práctica de la realidad, elevar la actitud consciente y por último lograr la conducta comprometida de estos con las demandas profesionales y sociales, para ello se utilizaron los métodos de persuasión, ejercitación, estímulo y amonestación (Martínez et al., 2003). La aplicación de uno u otro viene determinada por el contenido, los objetivos y el contexto en el cual se realiza la acción y actividad, teniendo como vía el diálogo, la conversación heurística, el debate, la confrontación de ideas, la búsqueda de información y la solución de problemas sociales, científicos entre otros.

Las acciones educativas propuestas tienen carácter curricular y extracurriculares responden a los procesos académicos, extensionistas y laborales que de conjunto y en las distintas formas asumidas se organizan de manera individual y/o colectiva, brindando un sistema de influencias formativas (Álvarez de Zayas, 1995). Para una mejor comprensión se estructura la propuesta en tres formas fundamentales de trabajo: teóricas, prácticas y de contacto visual. Es necesario reconocer que una sola forma por muy buena que esta sea, no puede resolver todas las necesidades educativas, las variantes tuvieron en cuenta el contexto, el tiempo y el diagnóstico de la realidad tratando de ser posible relacionar unas con otras.

Las formas verbales y de intercambio de ideas (teóricas) propuestas fueron resúmenes de lecturas a materiales dirigidos; síntesis de textos bibliográficos, revistas, periódicos; reflexiones a materiales audiovisuales $y$ elaboración de textos e ideas desde ambas asignaturas a través de los seminarios, clases prácticas, trabajos independientes, trabajos de cursos e integradores, en las asambleas de brigadas, arreglos de murales, boletines, eventos científicos, etc.

Las formas prácticas mediante las tareas y acciones realizables en la práctica docente interna y las acciones extensionistas curriculares y extracurriculares programadas de caminatas, excursiones, competencias, concursos, brigadas universitarias de trabajo social, sociedades científicas estudiantiles, trabajos voluntarios, festivales juveniles, encuentros de conocimientos, campañas de higienización, bastión universitario y días de la defensa, entre otras (Ivanovich, 1980)

Por último las formas visuales y de contacto social mediante los encuentros con personalidades de la profesión e históricas, visitas a lugares patrimoniales, observaciones y debates de videos audiovisuales y de registro de imágenes y sonidos.

Somos del criterio que ninguna propuesta de métodos y medios puede considerarse permanente, por lo que en cada contexto, según las características de los grupos, colectivos e individuos estos cambian según las exigencias y urgencias de los problemas, cada colectivo pedagógico propone sus propios medios, métodos y formas con la creatividad que los caracteriza y según las necesidades de sus educandos.

\section{RESULTADOS}

Más del $50 \%$ de los estudiantes muestran mayor protagonismo en clase y en las actividades extensionistas y de la FEU.

El $75 \%$ de los estudiantes participan en los debates y emiten juicios de valor desde el diálogo.

Manifiestan comportamientos responsable con la entrega a tiempo y en forma de los trabajos extra clase e investigativos el $90 \%$

Se involucran y forman parte de los círculos científicos estudiantiles más del $45 \%$.

Volumen 3 | número 1 | enero-junio | 2020 
Sociedad \& Tecnología | Revista del Instituto Tecnológico Superior Jubones | ISSN:

Incrementa en más del $92 \%$ la percepción del ejemplo personal del docente como paradigma en los estudiantes.

\section{CONCLUSIONES}

El trabajo educativo en la Carrera de Cultura Física ha logrado mediante el nexo afectivo profesor -alumno el sistema de influencias en valores dirigidos al trabajo formativo, la misma rechaza las manifestaciones de apatía, indisciplina de los estudiantes en los diferentes espacios educativos intra y extramuros; tiene como máxima el orientar al estudiante hacia un comportamiento responsable a partir de hacer suyos las normas, cualidades y valores sociales más relevantes del proyecto social cubano, desde la comprensión, valoración del papel que le corresponde jugar en la sociedad y el cumplimiento de tareas académicas, investigativas y laborales.

\section{REFERENCIAS BIBLIOGRÁFICAS}

Álvarez de Zayas, C. M. (1995). La pedagogía como ciencia (epistemología de la Educación). Editorial: Pueblo y Educación. Cuba. p. 14.

Báxter Pérez, E. (1998): La Educación en valores. Papel de la escuela. Documento del ICCP. Cuba.

Horruitiner Silva, P. (2003). La universidad cubana: el modelo de formación.

(2006): El proceso de formación en la universidad cubana. Pedagogía Universitaria. XI (3).

Ivanovich Boldiriev, N. (1980). Apuntes al trabajo educativo, editorial Moscú, p. 15-17.

Jiménez Padilla, T., \& Peralta Cuellar, JA. (2014) Reflexiones acerca del trabajo educativo actual en la enseñanza universitaria: premisa para la formación integral de los estudiantes de Cultura Física de la República de Cuba. Revista Digital EFDeportes.com. (190). Buenos Aires. http://www.efdeportes.com.

Martínez Llantada, M., Addine Fernández, F., García Legua, M., Martínez Angulo, M.,
Cruells Hernández, M.D. y Chiong Molina, M.O. (2004). Reflexiones teórico-prácticas desde las ciencias de la Educación. Editorial: Pueblo y Educación. Ciudad de La Habana. p. 51.

Palos Rodríguez, J. (1998). Educar para el futuro: temas transversales del curriculum. Editorial: Desclée De Brouwer. Bilbao. España.

Pérez Lemus, L. (2001). Diferentes enfoques en su proyección hacia el nuevo milenio. Revista Educación. (103). Editorial: Pueblo y Educación. p. 31. Cuba

Rojas Hernández, M. (2015). La labor educativa del docente universitario: un reto desde su función orientadora. Revista Pedagogía y Sociedad. 18(43). ISSN: 1608 - 3784. Cuba. 